\title{
A CRITICAL INDEX ALGORITHM FOR NEAREST POINT PROBLEMS ON SIMPLICIAL CONES*
}

\author{
Katta G. MURTY \\ Department of Industrial and Operations Engineering, University of Michigan, Ann Arbor, MI \\ 48019 , U.S.A.

\section{Yahya FATHI} \\ School of Industrial and Systems Engineering, Georgia Institute of Technology, Atlanta, GA \\ 30332, U.S.A.
}

Received 6 May 1980

Revised manuscript received 7 July 1981

\begin{abstract}
We consider the linear complementarity problem $(q, M)$ in which $M$ is a positive definite symmetric matrix of order $n$. This problem is equivalent to a nearest point problem $[\Gamma ; b]$ in which $\Gamma=\left\{A_{1}, \ldots, A \cdot n\right\}$ is a basis for $\mathbf{R}^{n}, b$ is a given point in $\mathbf{R}^{n}$; and it is required to find the nearest point in the simplicial cone $\operatorname{Pos}(\Gamma)$ to $b$. We develop an algorithm for solving the linear complementarity problem $(q, M)$ or the equivalent nearest point problem $[\Gamma ; b]$. Computational experience in comparison with an existing algorithm is presented.
\end{abstract}

Key words: Simplicial Cone, Nearest Point, Linear Complementarity Problem, Orthogonal Projection, Critical Index, Dimension Reduction.

\section{Introduction}

Let $\Gamma=\left\{A_{\cdot n}, \ldots, A_{\cdot n}\right\}$ be a given linearly independent set of column vectors in $\mathbf{R}^{n}$ and let $b \in \mathbf{R}^{n}$ be another given column vector. So $\Gamma$ is a basis for $\mathbf{R}^{n}$. Let $N=\{1, \ldots, n\}$ and let $A$ denote the square matrix consisting of $A_{._{1}}, \ldots, A_{\cdot n}$ as its columns. Let $\operatorname{Pos}(\Gamma)=\left\{x: x=A \alpha, \alpha=\left(\alpha_{1}, \ldots, \alpha_{n}\right)^{\mathrm{T}} \geqq 0\right\}$. For $x \in \operatorname{Pos}(\Gamma), \alpha=$ $A^{-1} x$ is known as the combination vector corresponding to $x$. We consider the problem of finding the nearest point (in terms of the usual Euclidean distance) in the simplicial cone $\operatorname{Pos}(\Gamma)$ to $b$. This problem will be denoted by the symbol $[\Gamma ; b]$ or $[A ; b]$, and will be called a nearest point problem of order $n$. The optimum solution of this problem is unique, and if $b \notin \operatorname{Pos}(\Gamma)$, then the solution lies on the boundary of $\operatorname{Pos}(\Gamma)$. If this point is $x^{*}$, then $\alpha^{*}=A^{-1} x^{*}$ is known as the optimum combination vector for $[\Gamma ; b]$. This problem is equivalent to the

\footnotetext{
* Research effort partially supported by the Air Force Office of Scientific Research. Air Force Systems Command, USAF, under grant No. AFOSR 78-3646. The United States Government is authorized to reproduce and distribute reprints for governmental purposes, not withstanding any copyright notation hereon.
} 
quadratic program

$$
\begin{array}{ll}
\text { Minimize } & -b^{\mathrm{T}} A \alpha+\frac{1}{2} \alpha^{\mathrm{T}}\left(A^{\mathrm{T}} A\right) \alpha, \\
\text { subject to } & \alpha=\left(\alpha_{1}, \ldots, \alpha_{n}\right)^{\mathrm{T}} \geqq 0 .
\end{array}
$$

The solution to (1) can be obtained by solving the following linear complementarity problem

$$
\begin{aligned}
& u-\left(A^{\mathrm{T}} A\right) \alpha=-A^{\mathrm{T}} b, \\
& u \geqq 0, \quad \alpha \geqq 0, \quad u^{\mathrm{T}} \alpha=0
\end{aligned}
$$

where $u=\left(u_{1}, \ldots, u_{n}\right)^{\mathrm{T}}$ is a column vector of variables in $\mathrm{R}^{n}$. Let $D=A^{\mathrm{T}} A$. Since $A$ is nonsingular, $D$ is positive definite and hence a $P$-matrix. The linear complementarity problem (2) has a unique complementary solution and if this solution is $\left(u^{*}, \alpha^{*}\right)$, then $\alpha^{*}$ is the optimum solution for (1), and hence the optimum combination vector for the nearest point problem $[A ; b]$. Also consider the following linear complementarity problem

$$
\begin{aligned}
& w-M z=q, \\
& w \geqq 0, \quad z \geqq 0, \quad w^{\mathrm{T}} z=0
\end{aligned}
$$

where $M$ is a positive definite symmetric matrix of order $n$. Let $L$ be a nonsingular matrix such that $L^{\mathrm{T}} L=M$ (for example, the Cholesky factor of $M$ ). See $[1,2]$ for efficient methods for computing the Cholesky factor. Now using earlier results, we conclude that if $\left(w^{*}, z^{*}\right)$ is the unique solution of (3), then $z^{*}$ is the optimum combination vector for the nearest point problem $\left[L ;-\left(L^{-1}\right)^{\mathrm{T}} q\right]$. Conversely if $z^{*}$ is the optimum combination vector for the nearest point problem $\left[L ;-\left(L^{-1}\right)^{\mathrm{T}} q\right]$, then $\left(w^{*}=M z^{*}+q, z^{*}\right)$ is the unique solution of (3). This clearly establishes that corresponding to each nearest point problem, there is an equivalent linear complementarity problem associated with a positive definite symmetric matrix and vice versa. This equivalence between the two problems is used to develop an algorithm for solving them, discussed in Section 2. Then in Section 3, the results of some numerical experiments with this algorithm are presented.

\section{A new algorithm}

In this section $(q, M)$ denotes the linear complementarity problem (3), where $M$ is a positive definite symmetric matrix of order $n$. Let $A$ denote a square nonsingular matrix of order $n$ satisfying $A^{\mathrm{T}} A=M$, and let $b=-\left(A^{-1}\right)^{\mathrm{T}} q$ and $\Gamma=\left\{A_{\cdot 1}, \ldots, A_{\cdot n}\right\}$. Then $[\Gamma ; b]$ is the nearest point problem equivalent to the linear complementarity problem $(q, M)$. The algorithm discussed here works with both of these problems. Let $\alpha^{*}$ be the unknown optimum combination 
vector for $[\Gamma ; b]$. Let $N=\{1, \ldots, n\}$ and $J \subset N$ be such that $J=\{j: \alpha\}>0\} . J$ is called the set of critical indices for the linear complementarity problem $(q, M)$ and for the corresponding nearest point problem $[\Gamma ; b]$. By our discussion in Section 1 , it is clear that the set of critical indices is also the set of all $j$ such that $z_{j}$ is strictly positive in the unique solution of $(q, M)$.

It is well known [5] that the solution to the nearest point problem $[\Gamma ; b]$ is the orthogonal projection of $b$ on the linear hull of $\left\{A_{j}: j \in J\right\}$. Hence if the set of critical indices is known, the solution to the nearest point problem $[\Gamma ; b]$ and correspondingly the solution to the associated linear complementarity problem $(q, M)$, is readily calculated. This fact can also be argued algebraically, directly. Notice that if $\left(w^{*}, z^{*}\right)$ is the unique solution of the linear complementarity problem $(q, M)$, then $w_{\mathrm{j}}^{*}=0$ for all critical indices $\mathrm{j}$, and $z_{\mathrm{j}}^{*}=0$ for all noncritical indices $j$; or equivalently if we define the vector $y=\left(y_{1}, \ldots, y_{n}\right)$ by

$$
\begin{aligned}
y_{j}=w_{j} & \text { for all } j \notin J, \\
z_{j} & \text { for all } j \in J,
\end{aligned}
$$

then $y$ is a complementary feasible basic vector for $(q, M)$, and $\left(w^{*}, z^{*}\right)$ is the basic solution of (3) corresponding to this basic vector $y$.

Even if a single critical index is known, this information can be used to reduce $(q, M)$ to a linear complementarity problem of order $n-1$ as shown in the following lemma.

Lemma 1. If a single critical index is known, $(q, M)$ can be reduced to a linear complementarity problem of order $n-1$.

Proof. Without loss of generality suppose we know that 1 is a critical index. Then perform a single principal pivot step in (3) in position 1. Suppose this leads to the system

\begin{tabular}{cccccccc|c|}
$w_{1}$ & $w_{2}$ & $\ldots$ & $w_{n}$ & $z_{1}$ & $z_{2}$ & $\ldots$ & $z_{n}$ & \\
\hline$-\bar{m}_{11}$ & 0 & $\ldots$ & 0 & 1 & $-\bar{m}_{12}$ & $\ldots$ & $\bar{m}_{1 n}$ & $\bar{q}_{1}$ \\
$-\bar{m}_{21}$ & 1 & $\ldots$ & 0 & 0 & $-\bar{m}_{22}$ & $\ldots$ & $-\bar{m}_{2 n}$ & $\bar{q}_{2}$ \\
$\vdots$ & $\vdots$ & $\vdots$ & $\vdots$ & $\vdots$ & $\vdots$ & $\vdots$ & $\vdots$ & $\vdots$ \\
$-\bar{m}_{n 1}$ & 0 & $\ldots$ & 1 & 0 & $-\bar{m}_{n 2}$ & $\ldots$ & $-\bar{m}_{n n}$ & $\bar{q}_{n}$ \\
\hline
\end{tabular}

Let $\bar{M}=\left(\bar{m}_{i j}: 2 \leqq i, j \leqq n\right)$ be the matrix of order $n-1$, and $\bar{q}=\left(\bar{q}_{2}, \ldots, \bar{q}_{n}\right)^{\mathrm{T}}$, from (5). Eliminating the columns of $w_{1}, z_{1}$, and the first row from (5) leads to the principal subproblem in variables $\omega=\left(w_{2}, \ldots, w_{n}\right)$ and $\xi=\left(z_{2}, \ldots, z_{n}\right)$, which is a linear complementarity problem of order $n-1$, denoted by $(\bar{q}, \bar{M})$. Since $M$ is positive definite and symmetric, so is $\overline{\mathcal{M}}$. If $\left(y_{2}, \ldots, y_{n}\right)$, where $y_{\mathrm{j}} \in\left\{w_{\mathrm{j}}, z_{\mathrm{j}}\right\}$, is a complementary feasible basic vector for $(\bar{q}, \bar{M})$, then, since $1 \in J,\left(z_{1}, y_{2}, \ldots, y_{n}\right)$ is 
a complementary feasible basic vector for the original $(q, M)$. Thus to solve $(q, M)$, if we know that $1 \in J$, it is enough if we solve the principal subproblem $(\bar{q}, \bar{M})$ of order $n-1$. Therefore the fact that $1 \in J$, has made it possible for us to reduce the linear complementarity problem $(q, M)$ of order $n$, into $(\bar{q}, \bar{M})$ of order $n-1$.

We can also argue geometrically that the knowledge of a critical index reduces the dimensionality of the nearest point problem. If 1 is a critical index, then the nearest point to $b$ in $\operatorname{Pos}(\Gamma)$ is also the nearest point to $b$ in $\operatorname{Pos}\left(\Gamma \cup\left\{-A_{.1}\right\}\right)$.

Define

$$
\begin{aligned}
& \bar{b}=b-A_{\cdot 1}\left(A_{\cdot 1}^{\mathrm{T}} b\right) /\left\|A \cdot{ }_{1}\right\|^{2}, \\
& \bar{A}_{\cdot j}=A \cdot{ }_{\cdot j}-A \cdot{ }_{1}\left(A_{\cdot 1}^{\mathrm{T}} A_{\cdot j}\right) /\|A \cdot\|^{2}, \quad \text { for } j=2, \ldots, n .
\end{aligned}
$$

Let $\left.\bar{\Gamma}=\left\{\bar{A}_{2}, \ldots, \bar{A}_{n}\right\}\right)$. For $2 \leqq j \leqq n, \bar{A}_{j}$ is orthogonal to $A_{1}$ and the cone $\operatorname{Pos}\left(\Gamma \cup\left\{-A_{1}\right\}\right)$ is the direct sum of the full line generated by $A_{\cdot 1}$ and the simplicial cone $\operatorname{Pos}(\bar{\Gamma})$. Solving $[\bar{\Gamma} ; \bar{b}]$ is an $(n-1)$ dimensional nearest point problem. If $\bar{x}^{*}$ is its solution, as embedded in $\mathbf{R}^{n}$, then $x^{*}$ defined by

$$
x^{*}=\bar{x}^{*}+A \cdot{ }_{1}\left(A_{\cdot 1}^{\mathrm{T}} b\right) /\left\|A \cdot{ }_{1}\right\|^{2}
$$

solves $[\Gamma ; b]$.

We will now develop an algorithm for finding a critical index. When it is obtained, we can reduce $(q, M)$ into a linear complementarity problem of lower order and apply the same approach on it.

\subsection{Definitions and notation}

Let $K_{j}$ denote the facet $\operatorname{Pos}\left(A_{1_{1}}, \ldots, A_{\cdot j-1}, A_{\cdot+1}, \ldots, A_{n}\right)$ of $\operatorname{Pos}(\Gamma)$ for $j \in N$. Let $x=\alpha_{1} A_{1_{1}}+\cdots+\alpha_{N} A_{n_{n}} \in \operatorname{Pos}(\Gamma)$. It follows that $\alpha_{j}=0$ if and only if $x \in K_{j}$, and $\alpha_{j}>0$ if and only if $x \notin K_{j}$, for all $j \in N$. Given the two points $b \in \mathbf{R}^{n}$ and $\bar{x} \in \mathbf{R}^{n}$ such that $b \neq \bar{x}$, let the open sphere $B(b ; \bar{x})=\{x:\|b-x\|<\|b-\bar{x}\|\}$. Consider the hyperplane $T(b ; \bar{x})=\left\{x:(x-\bar{x})^{\mathrm{T}}(b-\bar{x})=0\right\}$. The open half space $\left\{x:(x-\bar{x})^{\mathrm{T}}(b-\bar{x})>0\right\}$ is called the near side of $T(b ; \bar{x})$, while the closed half space $\left\{x:(x-\bar{x})^{\mathrm{T}}(b-\bar{x}) \leqq 0\right\}$ is called the far side of $T(b ; \bar{x})$. If the point $\bar{x}$ is chosen such that $0 \in T(b ; \bar{x})$, then $\tilde{x}^{\mathrm{T}}(b-\bar{x})=0$ and therefore for such $\bar{x}$ we have

$$
\begin{aligned}
& T(b ; \bar{x})=\left\{x: x^{\mathrm{T}}(b-\bar{x})=0\right\}, \\
& \text { Near side of } T(b ; \bar{x})=\left\{x: x^{\mathrm{T}}(b-\bar{x})>0\right\}, \\
& \text { Far side of } T(b ; \bar{x})=\left\{x: x^{\mathrm{T}}(b-\bar{x}) \leqq 0\right\} .
\end{aligned}
$$

For points $\bar{x}$ satisfying $0 \in T(b ; \bar{x})$, we define the set $N(\bar{x})$ by

$$
N(\bar{x})=\left\{j ; j \in N \text { and } A_{\cdot j}^{\top}(b-\bar{x})>0\right\} .
$$


So $N(\bar{x})$ is the set of subscripts of the column vectors in $\Gamma$ which are on the near side of $T(b ; \bar{x})$.

Let $\emptyset \neq S \subset \Gamma$. Define

$A(S)=$ Matrix with column vectors $A_{\cdot j} \in S$,

$I(S)=\{\mathrm{j}: A \cdot \mathrm{j} \in S\}$,

$\overline{I(S)}=N-I(S)$,

$H(S)=$ linear hull of $S$,

$b(S)=A(S)\left(A(S)^{\mathrm{T}} A(S)\right)^{-1} A(S)^{\mathrm{T}} b$, the orthogonal projection of $b$ onto $H(S)$

$\operatorname{Pos}(S)=$ the nonnegative hull of $S$.

$\operatorname{Pos}(S)$, which is a face of $\operatorname{Pos}(\Gamma)$, is called a projection face if $b(S) \in \operatorname{Pos}(S)$.

Let $V^{\mathrm{j}}=0$ if $A_{\cdot j}^{\mathrm{T}} b \leqq 0$, or $=A_{\cdot j}\left(A_{\cdot j}^{\mathrm{T}} b\right) /\|A \cdot\|_{\cdot j} \|^{2}$ if $A_{\cdot j}^{\mathrm{T}} b>0 . V^{\mathrm{j}}$ is the nearest point on the ray of $A_{\cdot j}$ to $b$. Also let $l \in N$ be such that $\left\|V^{l}-b\right\|=$ minimum $\left\{\left\|V^{j}-b\right\|: \mathrm{j} \in N\right\}$. Break ties for the minimum in this equation arbitrarily. Notice that if $V^{l} \neq 0$, it is the orthogonal projection of $b$ on the linear hull of $A .1$.

\subsection{Some results}

Consider the nearest point problem $[\Gamma ; b]$ and assume that $b \notin \operatorname{Pos}(\Gamma)$. The following results will be used in the development of the algorithm.

Lemma 2. If $V^{l}=0$ (or equivalently, if $b$ is in the polar cone of $\operatorname{Pos}(\Gamma)$ ), the nearest point in $\operatorname{Pos}(\Gamma)$ to $\mathrm{b}$ is 0 .

Proof. In this case $A_{\cdot j}^{\mathrm{T}} b \leqq 0$ for all $j \in N$. Hence the hyperplane $\left\{x: b^{\mathrm{T}} x=0\right\}$ for which the ray of $b$ is the normal at 0 , separates $b$ and $\operatorname{Pos}(\Gamma)$. So 0 is the nearest point in $\operatorname{Pos}(\Gamma)$ to $b$.

So, in the sequel, we assume that $V^{l} \neq 0$.

Lemma 3. A point $\bar{x} \in \operatorname{Pos}(\Gamma)$ is the nearest point in $\operatorname{Pos}(\Gamma)$ to $b$ if and only if

$$
0 \in T(b ; \bar{x}) \text { and } A_{\cdot j}^{\mathrm{T}}(b-\bar{x}) \leqq 0, \quad \text { for all } \mathrm{j} \in N
$$

Proof. Let $\bar{\alpha}=A^{-1} \bar{x}$. This theorem follows by (2) and the fact that the KarushKuhn-Tucker optimality conditions are both necessary and sufficient for (1) with $\bar{x}=A \bar{\alpha} \in \operatorname{Pos}(\Gamma)$ and $\bar{u}=-A^{\mathrm{T}}(b-\bar{x}) \geqq 0$.

Lemma 4. Let $\emptyset \neq S \subset \Gamma$ be such that $\bar{x}=b(S) \in \operatorname{Pos}(S)$. Then $0 \in T(b ; \bar{x})$. Also, in this case, if $N(\bar{x}) \cap \overline{I(S)}=\emptyset$, then $N(\bar{x})=\emptyset$, and $\bar{x}$ is the nearest point in $\operatorname{Pos}(\Gamma)$ to $b$.

${ }^{1}$ If $F$ and $G$ are any two sets, $F \backslash G$ denotes the set of all elements of $F$ which are not in $G$. 
Proof. Under the hypothesis $T(b ; \bar{x})$ contains $H(S)$ and hence $0 \in T(b ; \bar{x})$. Also, by the properties of orthogonal projection, the line joining $b$ and $\bar{x}$ is orthogonal to $H(S)$, and hence $(b-\bar{x})^{\mathrm{T}} A_{\cdot j}=0$ for all $\mathrm{j} \in I(S)$. So $N(\bar{x}) \cap \overline{I(S)}=\emptyset$ implies $N(\bar{x})=\emptyset$ in this case. By Lemma 3 these facts imply that $\bar{x}$ is the nearest point in $\operatorname{Pos}(\Gamma)$ to $b$.

Lemma 5. Let $\bar{x} \in \operatorname{Pos}(\Gamma)$ be such that $0 \in T(b ; \bar{x})$. If there exists an index $j \in N$ such that $A_{i}^{\mathrm{T}}(b-\bar{x}) \leqq 0$ for all $i \in N-\{j\}$, then $K_{i} \cap B(b ; \bar{x})=\emptyset$.

Proof. Clearly under these conditions $x^{\mathrm{T}}(b-\bar{x}) \leqq 0$ for all $x \in K_{i}$; however $x^{\mathrm{T}}(b-\bar{x})>0$ for all $x \in B(b ; \bar{x})$. Hence $K_{j} \cap B(b ; \bar{x})=\emptyset$.

Theorem 1. Let $\bar{x} \in \operatorname{Pos}(\Gamma)$ be such that $0 \in T(b ; \bar{x})$. If there exists an index $j \in N$ such that $A_{i}^{\mathrm{T}}(b-\bar{x}) \leqq 0$ for all $i \in N-\{j\}$ and $A_{\cdot j}^{\mathrm{T}}(b-\bar{x})>0$, then $j$ is a critical index of $[\Gamma ; b]$.

Proof. By Lemma 3, $\bar{x}$ is not the nearest point in $\operatorname{Pos}(\Gamma)$ to $b$. Let $\hat{x}$ be the nearest point in $\operatorname{Pos}(\Gamma)$ to $b$. Then $\hat{x} \in B(b ; \bar{x})$. By Lemma $5 K_{j} \cap B(b ; \bar{x})=\emptyset$. Hence $\hat{x} \notin K_{j}$ and thus $j$ is a critical index of $[\Gamma ; b]$.

Lemma 6. Given $0 \neq \bar{x} \in \operatorname{Pos}(\Gamma)$ satisfying $0 \in T(b ; \bar{x})$, if for some $i \in N$, we have

(i) $(b-\bar{x})^{\mathrm{T}} A_{\cdot i}>0$.

and either

(ii) $\|\bar{x}-b\| \leqq\left\|V^{i}-b\right\|$ and $\left\{\bar{x}, A_{\cdot}\right\}$ is linearly independent,

or

(ii) ${ }^{\prime} b^{\mathrm{T}} A_{\cdot i} \leq 0$,

then, the projection of $b$ onto the linear hull of $\left\{\bar{x}, A_{\cdot i}\right\}$ is in the relative interior of $\operatorname{Pos}\left\{\bar{x}, A_{i}\right\}$.

Proof. Since $\bar{x}$ is the closest point in $T(b ; \bar{x})$ to $b$ and since $0 \in T(b ; \bar{x}), \bar{x}$ is the closest point on the ray of $\bar{x}$ to $b$.

If (ii)' holds, then $V^{i}=0$ and hence in this case we have $\|\bar{x}-b\|<\left\|V^{i}-b\right\|$, and clearly $\left\{\bar{x}, A_{i}\right\}$ is linearly independent. So under these conditions (ii)' implies (ii).

By linear independence, $\operatorname{Pos}\{\bar{x}, A \cdot i\}$ is a two dimensional simplicial cone. Let $y$ be the closest point in $\operatorname{Pos}\left\{\bar{x}, A_{\cdot i}\right\}$ to $b$. By (i), $A_{\cdot i}$ is on the near side of $T(b ; \bar{x})$, and hence $B(b ; \bar{x}) \cap \operatorname{Pos}\left\{\bar{x}, A_{i}\right\} \neq \emptyset$. This implies that $y$ is closer than $\bar{x}$ to $b$; and by (ii), $y$ must be closer than $V^{i}$ to $b$. So $y$ is not contained on the rays of $\bar{x}$ or $A_{\text {.i }}$, and hence $y$ must be in the relative interior of $\operatorname{Pos}\left\{\tilde{\boldsymbol{x}}, \boldsymbol{A}_{i}\right\}$. 


\subsection{Routine for selecting a critical index}

This routine operates on the nearest point problem $[\Gamma ; b]$ which is equivalent to the given linear complementarity problem $(q, M)$. Clearly if $b \in \operatorname{Pos}(\Gamma)$, the nearest point in $\operatorname{Pos}(\Gamma)$ to $b$ is the point $b$ itself; so we assume that $b \notin \operatorname{Pos}(\Gamma)$ in the sequel. As mentioned earlier, we also assume that $V^{l} \neq 0$ (as otherwise, 0 is the nearest point in $\operatorname{Pos}(\Gamma)$ to $b)$.

The routine maintains a nonempty subset of $\Gamma$ called the current set denoted by $S$, and a point called the current point denoted by $\bar{x}, \bar{x} \in \operatorname{Pos}(S)$ always. As these things change from step to step, the symbols $S, \bar{x}$ may represent different things in different steps.

Initial step: Set $\bar{x}=V^{\prime}$, and compute $N(\bar{x})$. If $N(\bar{x})=\emptyset, \bar{x}$ is the nearest point in $\operatorname{Pos}(\Gamma)$ to $b$, terminate. If $N(\bar{x})$ is a singleton set, say $\left\{i_{1}\right\}, i_{1}$ is a critical index of $[\Gamma ; b]$, terminate this routine. If the cardinality of $N(\bar{x})$ is greater than or equal to 2, choose $g \in N(\bar{x})$; compute the orthogonal projection $\hat{b}$ of $b$ onto the linear hull of $\left\{\bar{x}, A_{. g}\right\}$. Replace $\bar{x}$ by $\hat{b}$. Set $S=\left\{A_{\cdot 1}, A_{g}\right\}$. Go to Step 1 .

Step 1: Let $S, \bar{x}$ be he current entities. Compute $N(\bar{x})$. If $N(\bar{x})=\emptyset, \bar{x}$ is the nearest point in $\operatorname{Pos}(\Gamma)$ to $b$, terminate. If $N(\bar{x})$ is a singleton set, say $\left\{i_{1}\right\}, i_{1}$ is a critical index of $[\Gamma, b]$, terminate this routine. If the cardinality of $N(\bar{x})$ is greater than or equal 2, go to Step 2 if $N(\bar{x}) \cap \overline{I(S)} \neq \emptyset$, or to Step 3 if $N(\bar{x}) \cap \overline{I(S)}=\emptyset$.

Step 2: Choose a $g \in N(\bar{x}) \cap \overline{I(S)}$. Compute $\hat{b}$, the orthogonal projection of $b$ onto the linear hull of $\{\bar{x}, A \cdot g\}$. Replace $S$ by $S \cup\left\{A_{g}\right\}$, and $\bar{x}$ by $\hat{b}$. Go back to Step 1.

Step 3: Compute $b(S)$. If $b(S) \in \operatorname{Pos}(S)$, replace $\bar{x}$ by $b(S)$ and go to Step 1. If $b(S) \notin \operatorname{Pos}(S)$, go to Step 4.

Step 4: Let the current point $\bar{x}=\sum\left(\alpha_{j} A_{\cdot j}: j \in I(S)\right)$, where $\alpha_{j} \geqq 0$ for all $j \in I(S)$. Let $b(S)=\sum\left[\beta_{\mathrm{j}} A_{\cdot j}: j \in I(S)\right]$. Since $b(S) \notin \operatorname{Pos}(S), \beta_{j}<0$ for some $\mathrm{j} \in I(S)$. An arbitrary point on the line segment joining $\bar{x}$ to $b(S)$ can be written as $Q(\lambda)=$ $(1-\lambda) \bar{x}+\lambda b(S), 0 \leqq \lambda \leqq 1$; or equivalently

$$
Q(\lambda)=\sum\left[\left((1-\lambda) \alpha_{j}+\lambda \beta_{j}\right) A_{\cdot j}: j \in I(S), 0 \leqq \lambda \leqq 1\right]
$$

As $\lambda$ increases from 0 to $1, Q(\lambda)$ moves from $\bar{x}$ to $b(S)$. Let $\lambda=\bar{\lambda}$ be the largest value of $\lambda$ for which $Q(\lambda)$ is in $\operatorname{Pos}(S)$. So $Q(\lambda)$ is on the boundary of $\operatorname{Pos}(S)$ and $Q(\lambda) \notin \operatorname{Pos}(S)$ for $\lambda>\vec{\lambda}$. So

$$
\bar{\lambda}=\operatorname{Max}\left\{\lambda:(1-\lambda) \alpha_{j}+\lambda \beta_{j} \geqq 0 \text { for all } \mathrm{j} \in I(S)\right\} .
$$

Let $k \in I(S)$ be such that $(1-\bar{\lambda}) \alpha_{k}+\bar{\lambda} \beta_{k}=0$. If there is more than one index in $I(S)$ with this property, choose one of them arbitrarily and call it $k . Q(\bar{\lambda})$ is the nearest point to $b(S)$ on the line segment joining $\bar{x}$ to $b(S)$ that lies in $\operatorname{Pos}(S)$. So $Q(\bar{\lambda}) \in \operatorname{Pos}\left(S<\left\{A_{\cdot k}\right\}\right)$. Delete $A_{\cdot k}$ from $S$. Also delete $k$ from $I(S)$ and include it in $\overline{I(S)}$. Replace $\bar{x}$ by $Q(\bar{\lambda})$ and go to Step 3 . 


\subsection{Discussion}

If termination does not occur in the initial step, when we move to Step 1 we will have $\|\bar{x}-b\|<\left\|V^{l}-b\right\|$ by Lemma 6 , and this property will continue to hold in all subsequent steps, since $\|\bar{x}-b\|$ never increases in the routine. Clearly $\bar{x} \in \operatorname{Pos}(S)$ always. These facts imply that once the algorithm enters Step 1, the cardinality of $S$ will always be greater than or equal to 2 .

While executing Step 4 , if $\bar{\lambda}$ turns out to be zero, there is no change in the point $\bar{x}$, but the cardinality of the set $S$ decreases by 1 at the end of this step. Thus a sequence of consecutive moves in the algorithm of the form Step $3 \rightarrow$ Step $4 \rightarrow$ Step $3 \cdots$, must terminate after at most $(n-2)$ visits to Step 4, with $\bar{x}$ set equal to $b(S)$ for some projection face $\operatorname{Pos}(S)$ in Step 3, and then the routine moves to Step 1 . When this happens, while executing Step 1, by Lemma 4 either the routine itself terminates; or else Step 2 must be taken implying a strict decrease in $\|\bar{x}-b\|$ by Lemma 6 with the new $\bar{x}$ via Step 2, and thus the projection face $\operatorname{Pos}(S)$ cannot repeat.

Whenever the routine visits Step 1 , the current point $\bar{x}$ is the orthogonal projection of $b$ onto a subspace of dimension 2 or more, and hence the property $0 \in T(b ; \bar{x})$ will hold then. Clearly, this property also holds in the initial step.

In the initial step, or in Step 1, if $N(\bar{x})=\emptyset, \bar{x}$ is the nearest point in $\operatorname{Pos}(\Gamma)$ to $b$ by Lemma 4 . In these steps, if $N(\bar{x})$ is a singleton set, the element in it is a critical index for $[\Gamma ; b]$ by Theorem 1 .

Since there are but a finite number of projection faces, these facts imply that if the routine does not terminate in the initial step, it terminates after a finite number of steps while executing Step 1.

When termination occurs in Step 1, it either finds the nearest point in $\operatorname{Pos}(\Gamma)$ to $b$, in which case the problem is completely solved, or it finds a critical index of the problem. In the latter case a linear complementarity problem of order $n-1$ can be constructed and the same routine can be applied to this smaller problem, as discussed in Lemma 1. The solution to the original problem then can be obtained using the solution of this smaller problem, as discussed in Lemma 1. Hence the unique solution of $(q, M)$ can be obtained after at most $n$ applications of the routine discussed above on linear complementarity problems of decreasing orders, each one associated with a positive definite symmetric matrix.

\section{Numerical experiment}

The experiments we report deal with the linear complementarity problem with a randomly generated data vector $q$ and a positive definite symmetric matrix $M$ with random entries $m_{i j}$. To assure that the matrix $M$ is positive definite and symmetric and that the problems were nontrivial, the following procedure was used: 
(a) Fill the matrix $A$ and the vector $b$ with random numbers which have a uniform distribution on the interval $[-0.5,+0.5]$, generated on the computer using the IMSL routines and rounded off to ten significant digits.

(b) Check whether or not $A$ is singular or $b \in \operatorname{Pos}(A)$. If so, discard the data and generate a new set of data.

(c) Let $M=A^{\mathrm{T}} A$ and $q=-A^{\mathrm{T}} b$. The matrix $M$ is now positive definite and symmetric.

We then solve the nearest point problem $[A ; b]$ which is equivalent to the linear complementarity problem $(q, M)$.

The purpose of these experiments is to compare the computational performance of the algorithm proposed by Wolfe in [7], with the algorithm discussed in Section 2. We refer to these algorithms as Algorithm I and Algorithm II respectively.

The codes for both algorithms were written in FORTRAN, using IMSL routines for matrix inversion and Cholesky factorization. Instead of using computer times for our comparisons, we count the number of iterations and compute the total number of multiplication and/or division operations required to do each of the problems, and compute the average over all the problems. The experiments consist of running one hundred randomly generated problems in each of the four different sizes $n=20,30,40$ and 50. The average work is then determined and presented in Table 1.

In view of these results, Algorithm II holds promise as being more efficient than Algorithm I, especially for problems of larger sizes.

Table 1

Numerical results of the experiments

\begin{tabular}{ccc}
\hline $\begin{array}{l}\text { Size of the } \\
\text { problem }(n)\end{array}$ & $\begin{array}{l}\text { Average number of multiplication and/or } \\
\text { division operations required to solve the } \\
\text { problem }\end{array}$ \\
\cline { 2 - 3 } & Algorithm I & Algorithm II \\
\hline 20 & 30069 & 16266 \\
30 & 123644 & 42592 \\
40 & 514822 & 170643 \\
50 & 896919 & 324126 \\
\hline
\end{tabular}

\section{Acknowledgment}

We are grateful to two unknown referees for a lot of excellent suggestions to improve the presentation throughout the paper. The geometric argument for Lemma 1 is due to one of the referees, and the delineation of the results in Section 2 and the finite termination argument in Section 3 benefited a lot from the insightful suggestions of referee 2 . 


\section{References}

[1] D.K. Faddev and V.N. Faddeeva, Computational methods of linear algebra (Freeman, San Francisco, CA, 1963).

[2] P.E. Gill, G.H. Golub, W. Murray and M.A. Saunders, "Methods for modifying matrix factorizations", Mathematics of Computation 28 (1974) 505-535.

[3] K.G. Murty, Linear and combinatorial programming (Wiley, New York, 1976).

[4] K.G. Murty, "On the number of solutions to the complementarity problem and spanning properties of complementary cones", Linear Algebra and Its Applications 5 (1972) 65-108.

[5] K.G. Murty, "On the linear complementarity problem", Operations Research Verfahren 31 (1978) 425-439.

[6] D.R. Wilhelmsen, "A nearest point algorithm for convex polyhedral cones and applications to positive linear approximation", Mathematics of Computation 30 (1976) 48-57.

[7] P. Wolfe, "Finding the nearest point in a polytope", Mathematical Programming 11 (1976) 128-149. 